\title{
Corneal Dose Reduction Using a Bismuth-Coated Latex Shield over the Eyes During Brain SPECT/CT
}

\author{
Norikazu Matsutomo ${ }^{1}$, Masaaki Fukunaga ${ }^{2}$, Hideo Onishi $^{3}$, and Tomoaki Yamamoto ${ }^{1}$ \\ ${ }^{1}$ Department of Medical Radiological Technology, Faculty of Health Sciences, Kyorin University, Tokyo, Japan; ${ }^{2}$ Department of \\ Radiological Technology, Kurashiki Central Hospital, Okayama, Japan; and ${ }^{3}$ Program in Biological System Sciences, Graduate \\ School of Comprehensive Scientific Research, Prefectural University of Hiroshima, Hiroshima, Japan
}

This study aimed to determine whether a bismuth-coated latex shield (B-shield) could protect the eyes during brain SPECT/CT. Methods: A shield containing the heavy metal bismuth (equivalent to a $0.15-\mathrm{mm}$-thick lead shield) was placed over a cylindric phantom and the eyes of a 3-dimensional brain phantom filled with $99 \mathrm{~m} T \mathrm{Tc}$ solution. Subsequently, phantoms with and without the B-shield were compared using SPECT/CT. The CT parameters were 30-200 mA and $130 \mathrm{kV}$. The dose reduction achieved by the B-shield was measured using a pencilshaped ionization chamber. The protective effects of the $\mathrm{B}$-shield were determined by evaluating relative radioactivity concentration as well as artifacts (changes in CT number), linear attenuation coefficients, and coefficients of variation on SPECT images. Results: The radiation doses with and without the Bshield were 0.14-0.77 and 0.36-1.93 mGy, respectively, and the $\mathrm{B}$-shield decreased the average radiation dose by about $60 \%$. The B-shield also increased the mean CT number, but only at locations just beneath the surface of the phantom. Streaks of higher density near the underside of the B-shield indicated beam hardening. Linear attenuation coefficients and the coefficients of variation did not significantly differ between phantoms with and without the B-shield, and the relative ${ }^{99 m T c}$ radioactivity concentrations were not affected. Conclusion: The B-shield decreased the radiation dose without affecting estimated attenuation correction or radioactivity concentrations. Although surface artifacts increased with the B-shield, the quality of the SPECT images was acceptable. B-shields can help protect pediatric patients and patients with eye diseases who undergo SPECT imaging.

Key Words: radiation dose; radiation shielding; image quality; SPECT/CT; attenuation correction

J Nucl Med Technol 2017; 45:214-218

DOI: 10.2967/jnmt.117.192849

\section{0} PCTCT is a medical imaging technology that combines SPECT and CT. The CT imaging provides accurate nonuniform attenuation correction for SPECT images of the brain when the position of the head is fixed in a holder.

\footnotetext{
Received Mar. 11, 2017; revision accepted Apr. 26, 2017.

For correspondence or reprints contact: Norikazu Matsutomo, Department of Medical Radiological Technology, Faculty of Health Sciences, Kyorin University, A-420, 5-4-1 Shimorenjaku Mitaka-shi, Tokyo, 181-8612, Japan. E-mail: nmatsutomo@ks.kyorin-u.ac.jp

Published online May 4, 2017.

COPYRIGHT (c) 2017 by the Society of Nuclear Medicine and Molecular Imaging.
}

Consequently, integrated SPECT/CT systems enable the collection of accurate qualitative and quantitative dopamine transporter images $(1,2)$. However, the $\mathrm{CT}$ procedure increases the radiation dose to the patient, especially to the eye lens, which is among the most radiosensitive tissues in humans (3). From this viewpoint, the International Commission on Radiological Protection has recommended a threshold of $500 \mathrm{mGy}$ for the dose absorbed by the eye lens $(4,5)$. Accordingly, various techniques have been used to reduce the radiation dose delivered by $\mathrm{CT}(6-8)$. Widely used approaches include tube current control and automated exposure control (9), iterative reconstruction (10), and shielding of organs (11). Although automated exposure control and iterative reconstruction have good potential to reduce the radiation dose while maintaining image quality, these are not available in all systems. On the other hand, superficial organs have been protected with bismuth-coated latex shields (B-shields) which have a reasonable cost and suitable attenuation characteristics. Hein et al. (12) reported that such shields can reduce the surface radiation dose by $40 \%$ during paranasal CT. In addition, Hopper et al. (13) reported that $\mathrm{B}$-shields can decrease the average radiation dose by $48.5 \%$. These findings indicate that B-shields might also be useful during SPECT/CT imaging. However, the effects of B-shields on brain SPECT/CT images have not been investigated, especially the accuracy of attenuation correction and SPECT image quality.

The purpose of this study was to assess whether Bshields can be used to protect the eyes during brain SPECT/ CT imaging. We evaluated the quality of brain SPECT/CT images acquired when a B-shield was used and the ability of the shield to reduce the radiation dose.

\section{MATERIALS AND METHODS}

\section{SPECT/CT Instrument, Phantoms, and B-Shield}

We acquired and reconstructed all images using a Symbia T2 dual-head hybrid SPECT/CT camera (Siemens AG) equipped with a low-energy, high-resolution collimator. The CT component is the same as that for the Sensation scanner (Siemens AG). The radiation dose was measured using a CT dose index phantom, and SPECT image quality was assessed using a cylindric phantom (diameter, $16 \mathrm{~cm}$; height, $15 \mathrm{~cm}$ ) and a 3-dimensional (3D) brain phantom (14) containing a solution of ${ }^{99 \mathrm{~m}} \mathrm{Tc}$ (activity, 32.8 or 45.8 $\mathrm{kBq} / \mathrm{mL}$ ). A in-house-developed flexible shield containing bismuth 


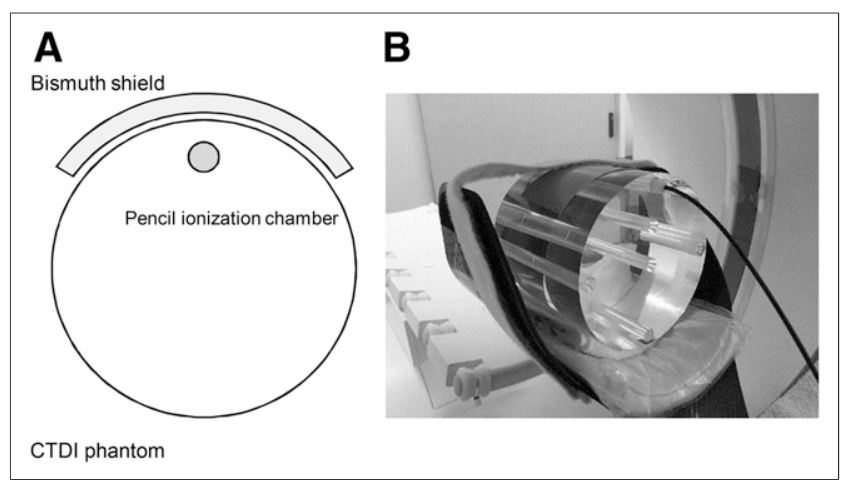

FIGURE 1. Phantom with B-shielding. (A) CT dose index (CTDI) phantom with B-shielding placed over surface. (B) Pencil-shaped ionization chamber positioning.

(equivalent to a 0.15 -mm-thick lead shield) was placed over the surface of the phantom and shaped to cover both eyes.

\section{Dose Measurements}

We measured the radiation dose at the top area of the CT dose index phantom with and without the B-shield (Fig. 1) using a pencil-shaped ionization chamber (Toyo Medical Co.). The eye lens equivalent dose should ideally be assessed at a depth of $3 \mathrm{~mm}$, but the value more commonly used is at a depth of $70 \mu \mathrm{m}$ or $1 \mathrm{~cm}$, and this is within $10 \%$ of the actual value. Therefore, the ionization chamber was inserted at a 1-cm depth in the CT dose index phantom. The phantom was assessed by CT using the parameters $130 \mathrm{kVp}, 30-200 \mathrm{~mA}, 2 \times 0.5-\mathrm{mm}$ collimation, 0.8 -s rotation time, 5 - $\mathrm{mm}$ slice thickness, and a pitch value of 0.95 . The radiation dose reduction caused by the B-shield was calculated as

$$
\text { Reduction }=\frac{\text { Dose }_{\text {cont }}-\text { Dose }_{\text {bismuth }}}{\text { Dose }_{\text {cont }}} \times 100(\%),
$$

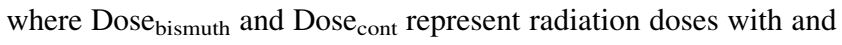
without the B-shield, respectively. The measurements were repeated 5 times for each CT scan.

\section{Evaluation of Image Quality}

Data Acquisition and Reconstruction. SPECT/CT data were acquired with and without the B-shield using both cylindric and $3 \mathrm{D}$ brain phantoms. Projection data were obtained in continuous mode with rotation through 120 angular views. The pixel size was $3.3 \times 3.3 \mathrm{~mm}$ (scale for enlargement, 1.45) with a $128 \times 128$ matrix. The total acquisition time was 20 min (10 repetitions of $2 \mathrm{~min} /$ cycle). A photopeak of ${ }^{99 \mathrm{~m}} \mathrm{Tc}$ was set as a $21 \%$ energy window centered at $140 \mathrm{keV}$. A low subwindow to correct multipleenergy-window scatter was set at $7 \%$ on the bottom of the photopeak window (116-124 keV). All CT images were acquired using the same parameters as in the dose measurement.

All phantom images were reconstructed using ordered-subset expectation maximization with resolution recovery, and both scatter correction and CT-based attenuation correction were derived from the current in the CT tube. The reconstruction parameters were 15 iterations, 6 subsets, and 3D gaussian filtering of $8.6 \mathrm{~mm}$ in full width at half maximum as postsmoothing.

Data Analysis. Five circular regions of interest (ROIs) were placed on the top, middle, and bottom of the CT images and the attenuation correction map (Fig. 2). The mean CT number and linear attenuation coefficients were then measured. The same ROIs were placed on the SPECT images, and the coefficient of variation (CV) was calculated as

$$
\mathrm{CV}=\frac{\mathrm{SD}}{\text { mean }} \times 100 \%
$$

where the mean represents the mean count in the SPECT image ROI and SD is the SD of the ROI. We also investigated the effect of the B-shield on regional radioactivity by automatically placing ROI templates on 3D stereotactic ROIs (15). The anterior, precentral, central, parietal, angular, temporal, occipital, pericallosal, lenticular, thalamic, hippocampal, and cerebellar regions were used in the analysis. The difference in regional radioactivity was calculated as

$$
\text { Difference }=\frac{\text { mean }_{\text {ref }}-\text { mean }_{\text {bismuth }}}{\text { mean }_{\text {ref }}} \times 100(\%),
$$

where mean $n_{\text {bismuth }}$ and mean ${ }_{\text {ref }}$ are the mean SPECT counts with and without B-shielding, respectively.

\section{Statistical Analysis}

All data were compared using a paired $t$ test, with a $P$ value of less than 0.05 indicating a statistically significant difference.

\section{RESULTS}

\section{Dose Measurement}

The radiation doses and reduction values are shown in Table 1 . The radiation dose was significantly lower $(60.3 \% \pm$ $0.5 \%$ ) with the B-shield at each tube current.

\section{Image Quality}

The mean CT number when using the B-shield was significantly increased at the top of the phantom (Fig. 3A) and slightly increased at the middle and bottom of the phantom (Fig. 3B). Figure 4 shows a comparison of linear attenuation coefficients with and without the B-shield. Although linear attenuation coefficients at the top of the phantom were increased when using the B-shield, changes could not be detected at the middle or bottom. The coefficient of variation of SPECT images with and without the B-shield are shown in Figure 5. The coefficients of variation were essentially constant, and no differences between the shielding and nonshielding conditions could be detected, especially at the middle or bottom of the phantom.

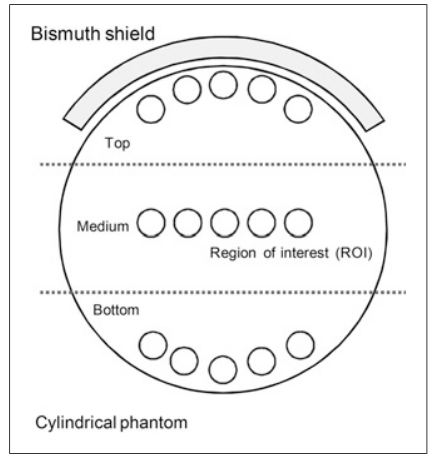

FIGURE 2. Images with ROI settings for evaluation. ROls are placed at top, middle, and bottom of image. 
TABLE 1

Effect of B-Shielding on Radiation Doses

\begin{tabular}{ccccccc}
\hline & \multicolumn{6}{c}{ Tube current (mA) } \\
\cline { 2 - 7 } Parameter & 30 & 40 & 50 & 100 & 150 & 200 \\
\hline $\begin{array}{c}\text { Dose without shield } \\
\text { (mGy) }\end{array}$ & 0.36 & 0.42 & 0.48 & 0.96 & 1.45 & 1.93 \\
$\begin{array}{c}\text { Dose with shield } \\
\text { (mGy) }\end{array}$ & 0.14 & 0.17 & 0.19 & 0.38 & 0.58 & 0.77 \\
Reduction (\%) & 61.1 & 59.5 & 60.4 & 60.4 & 60.0 & 60.1 \\
\hline
\end{tabular}

Figure 6 shows images acquired at tube currents of 50, 100 , and $200 \mathrm{~mA}$. For the 3D brain phantom, the radiation dose remained essentially the same with and without the $\mathrm{B}$-shield. The difference in the radioactivity concentrations with and without B-shielding was the largest in the thalamus and was no more than $5.1 \%$ in any other region. Figure 7 shows that radioactivity concentrations on SPECT images were similar with and without the B-shield.

\section{DISCUSSION}

Although particularly radiosensitive organs are conventionally protected with a lead shield during radiography, lead causes metal artifacts and decreases image quality. However, B-shields and conventional lead shields react differently to $\mathrm{x}$-rays. Lead shields completely absorb radiation, whereas B-shields decrease the amount of soft radiation and thus alter only the x-ray energy distribution. Therefore, B-shields should be useful for protecting radiosensitive organs, such as the eye lens, because B-shields generate fewer metal artifacts than lead shields.

The present study evaluated whether B-shields could reduce the radiation dose and maintain the accuracy of SPECT images. We found that B-shields reduced the radiation dose to the eye lens by about $60 \%$ and that radioactivity concentrations with and without the B-shield were equivalent. These

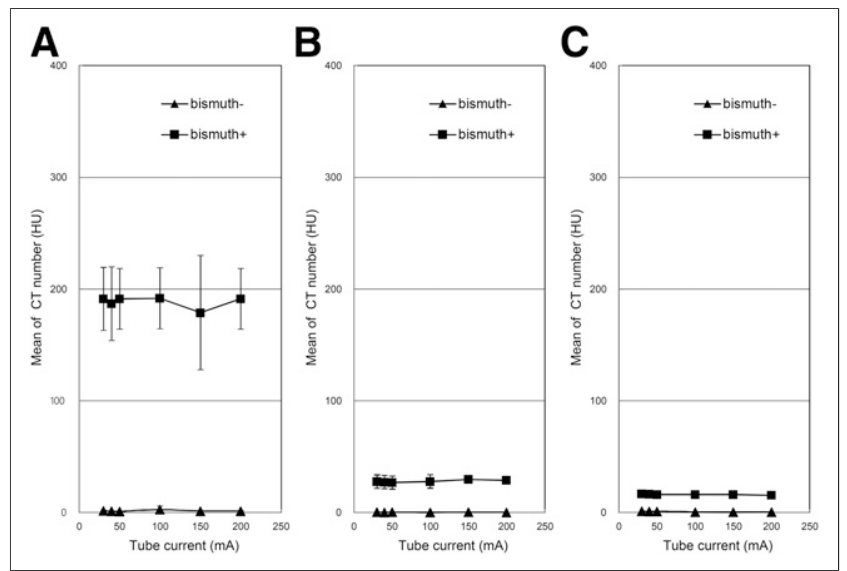

FIGURE 3. Mean of CT number determined on CT images as function of B-shielding at top (A), middle (B), and bottom (C) of image. Mean CT number generated with shielding is slightly higher at middle and bottom of image.

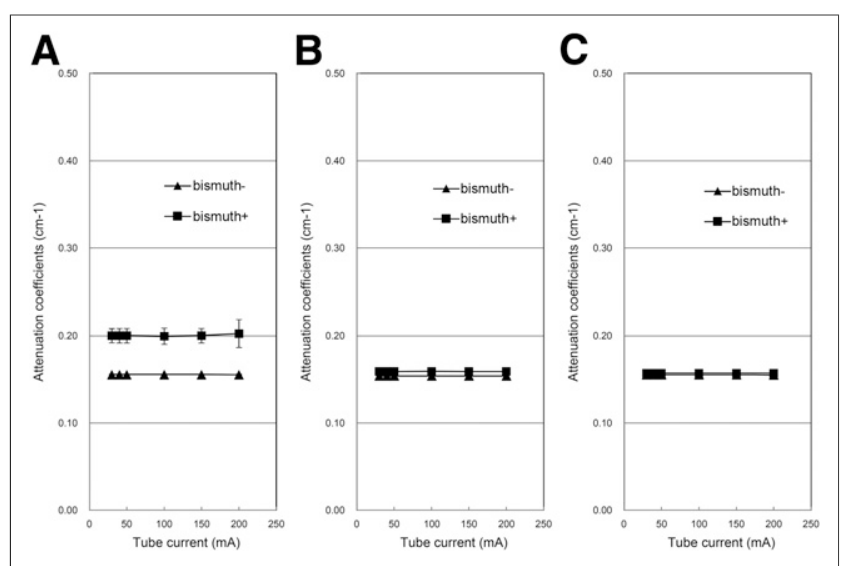

FIGURE 4. Comparison of attenuation coefficients with and without $B$-shielding at top $(A)$, middle $(B)$, and bottom (C) of image. Attenuation coefficients at middle and bottom of image are not significantly different with and without B-shielding.

results indicate that B-shields could confer radioprotection, especially for pediatric patients and patients with extant eye diseases who undergo brain SPECT/CT imaging.

The radiation dose was reduced by about $60 \%$ at the top of the CT dose index phantom at each tube current. For comparison, Ciarmatori et al. (16) reported that B-shielding can reduce the entrance surface dose by $21 \%-29 \%$ during head CT examinations and Wang et al. (17) reported that a $\mathrm{B}$-shield and tube current modulation reduced the radiation dose to the eye lens by $26.4 \%$. Our results are essentially consistent with these previous findings, but the dose reduction was significantly greater during $\mathrm{CT}$ imaging in the present study. The reason for this difference is the difference in the lead equivalent of the B-shield. The lead equivalent in the current and previous studies was 0.15 and $0.06 \mathrm{~mm}$, respectively. Hopper et al. (13) showed that the CT dose decreased when the shield thickness increased. Therefore, we think that optimization of the lead equivalent
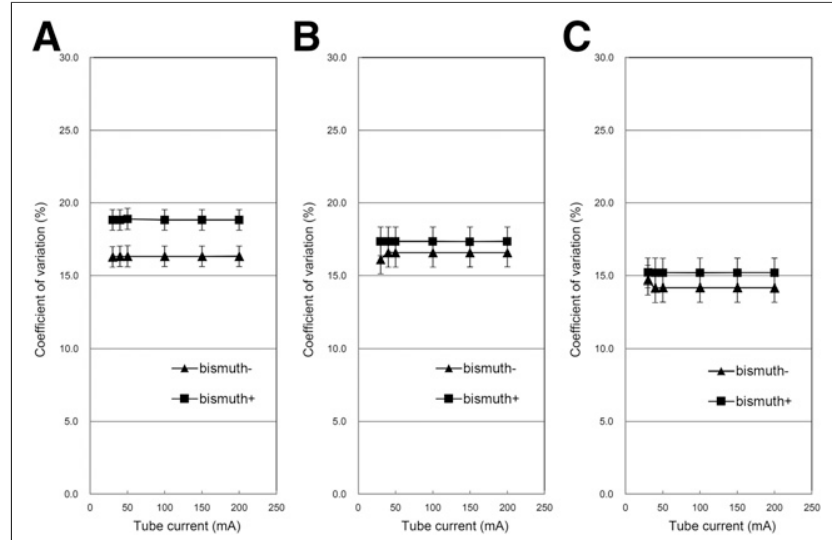

FIGURE 5. Coefficient of variation as function of B-shielding at top (A), middle (B), and bottom (C) of image. Coefficient of variation $=\mathrm{SD} /$ mean. 


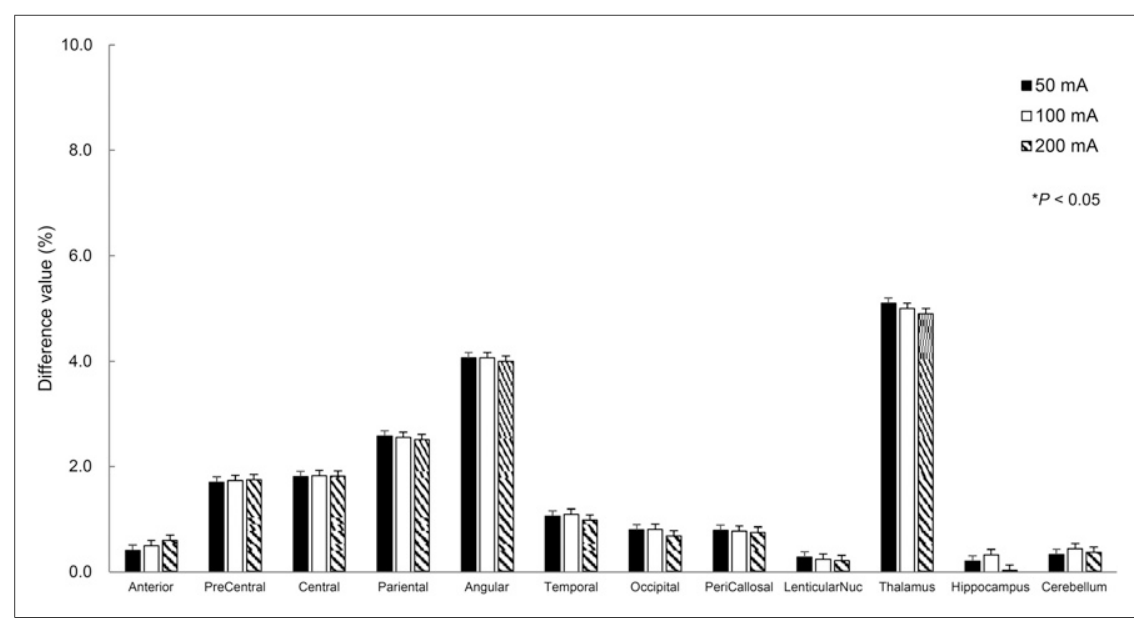

FIGURE 6. Comparison of regional radioactivity with and without $B$-shielding. Amounts of regional radioactivity do not differ significantly. Difference was highest in thalamus $(5.1 \%)$. is necessary for more effective dose reduction. Our results indicate that the CT dose per scan is tiny compared with the International Commission on Radiological Protectionrecommended dose $(<2.0 \mathrm{mGy})$. However, for radiation examinations, every effort should be made to reduce radiation doses without compromising image quality according the "as low as reasonably achievable" concept. The use of B-shielding is considered to improve the benefits of brain SPECT/CT for patients.

The mean CT number increased with the B-shield because of beam-hardening artifacts, but this increase was limited to a superficial region. This result agrees with those of previous studies. Hopper et al. (13) reported that the increased CT number caused by a protection shield did not project into the brain. Hein et al. (12) found that use of an eye shield did not impair diagnostic information in a study of 127 patients. In addition, Hohl et al. (18) showed that a spacer between the surface and shield can reduce the deleterious effects on image quality caused by beamhardening artifacts.

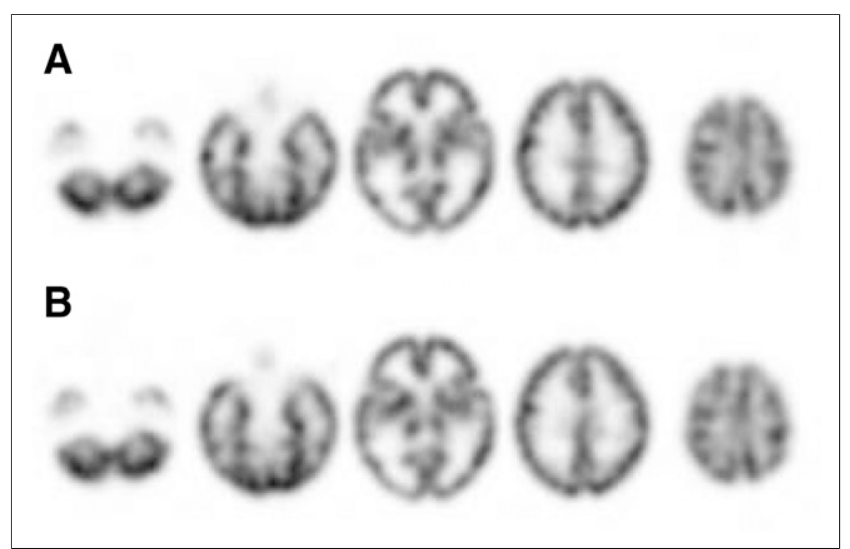

FIGURE 7. Examples of SPECT images acquired with Bshielding. (A) Reference image from 3D brain phantom. (B) Image acquired with B-shielding. Quality of both images is similar.
Linear attenuation coefficients did not significantly differ between the shielded and nonshielded conditions at the middle and bottom of the phantom. The linear attenuation coefficient was estimated on the basis of the CT number. Because the CT number changed only slightly, we considered that the accuracy of the attenuation coefficient was maintained when using the B-shield. We also found that the coefficient of variation was similar with and without the B-shield. These findings indicate that B-shields can be used during brain SPECT/CT image acquisition.

The relative amounts of radioactivity in the 3D brain phantom with and without the B-shield did not differ significantly. The maximum difference between measurements with and without B-shielding was $5.1 \%$, and this was found only in the thalamus. For comparison, Akamatsu et al. (19) reported that the regional radioactivity differed by $1.8 \%-11.5 \%$ among several reconstruction methods. Ishii et al. (20) showed that the relative SPECT counts obtained by Chang's attenuation correction increased by $5.0 \%$ and decreased by $4.6 \%$ in the frontal and cerebellar regions, respectively, compared with $\mathrm{CT}$ attenuation correction. The results support the notion that the use of a B-shield can benefit patients by decreasing the radiation exposure risk to their corneas. Although B-shields have several disadvantages, we recommend their application from the perspective of radiation protection during brain SPECT/CT.

This study has some limitations. One is the simple protocol using several geometric phantoms. In addition, the region of white matter in the $3 \mathrm{D}$ brain phantom did not contain the radioactive solution. Further study is required to evaluate clinical brain SPECT/CT with B-shields. Another limitation is that we did not compare the effect of dose reduction using $\mathrm{B}$-shields with dose reduction using other techniques, such as organ-based tube current modulation and CT iterative reconstruction $(21,22)$. These techniques can also potentially reduce the radiation dose. The effects of the B-shield combined with other methods of reducing the radiation dose should be evaluated. 


\section{CONCLUSION}

We evaluated the effects of a B-shield on brain SPECT/ CT images in a phantom study. The shield reduced the dose of radiation delivered to the eye lens by about $60 \%$. Although the B-shield increased image artifacts at the surface, the concentration of radioactivity was acceptable. Our results suggest that the B-shield can help protect patients undergoing assessment by brain SPECT/CT, especially pediatric patients and patients with eye disease.

\section{DISCLOSURE}

No potential conflict of interest relevant to this article was reported.

\section{REFERENCES}

1. Ueda J, Yoshimura H, Shimizu K, Hino M, Kohara N. Combined visual and semiquantitative assessment of ${ }^{123}$ I-FP-CIT SPECT for the diagnosis of dopaminergic neurodegenerative diseases. Neurol Sci. April 7, 2017 [Epub ahead of print].

2. Lapa C, Spehl TS, Brumberg J, et al. Influence of CT-based attenuation correction on dopamine transporter SPECT with ${ }^{123}$ IFP-CIT. Am J Nucl Med Mol Imaging. 2015;5:278-286.

3. Mountford PJ, Temperton DH. Recommendations of the International Commission on Radiological Protection (ICRP) 1990. Eur J Nucl Med. 1992; 19:77-79.

4. Statement on tissue reactions: approved by the commission on April 21, 2011 (ICRP ref 4825-3093-1464). U.S. Nuclear Regulatory Commission website. https://www.nrc.gov/docs/ML1326/ML13269A317.pdf. Accessed June 12, 2017.

5. Stewart FA, Akleyev AV, Hauer-Jensen M, et al. ICRP publication 118: ICRP statement on tissue reactions and early and late effects of radiation in normal tissues and organs - threshold doses for tissue reactions in a radiation protection context. Ann ICRP. 2012;41(1-2).

6. Heaney DE, Norvill CA. A comparison of reduction in CT dose through the use of gantry angulations or bismuth shields. Australas Phys Eng Sci Med. 2006;29:172-178.

7. Nikupaavo U, Kaasalainen T, Reijonen V, Ahonen SM, Kortesniemi M. Lens dose in routine head CT: comparison of different optimization methods with anthropomorphic phantoms. AJR. 2015;204:117-123.
8. Reimann AJ, Davison C, Bjarnason T, et al. Organ-based computed tomographic (CT) radiation dose reduction to the lenses: impact on image quality for CT of the head. J Comput Assist Tomogr. 2012;36:334-338.

9. Greess $\mathrm{H}$, Wolf $\mathrm{H}$, Baum U, et al. Dose reduction in computed tomography by attenuation-based on-line modulation of tube current: evaluation of six anatomical regions. Eur Radiol. 2000;10:391-394.

10. McLaughlin DJ, Mooney RB. Dose reduction to radiosensitive tissues in CT: do commercially available shields meet the users' needs? Clin Radiol. 2004;59:446450.

11. Perisinakis K, Raissaki M, Theocharopoulos N, Damilakis J, Gourtsoyiannis N. Reduction of eye lens radiation dose by orbital bismuth shielding in pediatric patients undergoing CT of the head: a Monte Carlo study. Med Phys. 2005;32:10241030 .

12. Hein E, Rogalla P, Klingebiel R, Hamm B. Low-dose CT of the paranasal sinuses with eye lens protection: effect on image quality and radiation dose. Eur Radiol. 2002;12:1693-1696.

13. Hopper KD, Neuman JD, King SH, Kunselman AR. Radioprotection to the eye during CT scanning. AJNR Am J Neuroradiol. 2001;22:1194-1198.

14. Iida $\mathrm{H}$, Hori $\mathrm{Y}$, Ishida $\mathrm{K}$, et al. Three-dimensional brain phantom containing bone and grey matter structures with a realistic head contour. Ann Nucl Med. 2013;27:25-36.

15. Takeuchi R, Yonekura Y, Matsuda H, Konishi J. Usefulness of a three-dimensional stereotaxic ROI template on anatomically standardised $99 \mathrm{~m}$ Tc-ECD SPECT. Eur J Nucl Med Mol Imaging. 2002;29:331-341.

16. Ciarmatori A, Nocetti L, Mistretta G, Zambelli G, Costi T. Reducing absorbed dose to eye lenses in head CT examinations: the effect of bismuth shielding. Australas Phys Eng Sci Med. 2016;39:583-589.

17. Wang J, Duan X, Christner JA, Leng S, Grant KL, McCollough CH. Bismuth shielding, organ-based tube current modulation, and global reduction of tube current for dose reduction to the eye at head CT. Radiology. 2012;262:191-198.

18. Hohl C, Wildberger JE, Süss C, et al. Radiation dose reduction to breast and thyroid during MDCT: effectiveness of an in-plane bismuth shield. Acta Radiol. 2006; 47:562-567.

19. Akamatsu M, Yamashita Y, Akamatsu G, et al. Influences of reconstruction and attenuation correction in brain SPECT images obtained by the hybrid SPECT/CT device: evaluation with a 3-dimensional brain phantom. Asia Ocean J Nucl Med Biol. 2014;2:24-29.

20. Ishii K, Hanaoka K, Okada M, et al. Impact of CT attenuation correction by SPECT/CT in brain perfusion images. Ann Nucl Med. 2012;26:241-247.

21. McCollough CH, Primak AN, Braun N, Kofler J, Yu L, Christner J. Strategies for reducing radiation dose in CT. Radiol Clin North Am. 2009;47:27-40.

22. Prakash P, Kalra MK, Kambadakone AK, et al. Reducing abdominal CT radiation dose with adaptive statistical iterative reconstruction technique. Invest Radiol. 2010;45:202-210. 